\title{
LHC Status and Performance
}

\author{
J. Wenninger* \\ CERN, Geneva, Switzerland \\ E-mail: Jorg.Wenninger@cern.ch
}

The CERN Large Hadron Collider operated during Run 2 (2015-2018) at a beam energy of 6.5 TeV. This period saw an important increase in performance through improvements in the LHC and an increased beam quality from its injectors, leading to a peak luminosity that exceeds the LHC design luminosity by more than a factor two. This contribution will give an overview of LHC Run 2 and the main challenges that were encountered. Finally an outlook will be given on the performance in Run 3 (2021-2023).

Prospects for Charged Higgs Discovery at Colliders - CHARGED2018

25-28 September 2018

Uppsala, Sweden

* Speaker. 


\section{Introduction}

The Large Hadron Collider at CERN, Geneva, is a $26.7 \mathrm{~km}$ long circular accelerator [1]. It is based on a superconducting two-in-one magnet design with a target beam energy of $7 \mathrm{TeV}$. It features 8 straight sections: 4 Interaction Regions (IRs) are reserved for accelerator equipment and 4 house particle physics experiments: the two high luminosity experiments ATLAS and CMS, the medium luminosity experiment LHCb and the low luminosity experiment ALICE.

The LHC was first operated with beam for short periods in 2008 and 2009. In 2010 a first experience with the machine was gained at a beam energy of $3.5 \mathrm{TeV}$, and moderate beam intensity (up to $\approx 200$ bunches of $1.1 \times 10^{11} \mathrm{p}$ per bunch, or ppb). In 2011 the beam intensity was pushed to $\approx 1400$ bunches of $1.4 \times 10^{11} \mathrm{ppb}$ while 2012 was dedicated to luminosity production with higher bunch intensities $\left(1.6 \times 10^{11} \mathrm{ppb}\right)$ and a beam energy of $4 \mathrm{TeV}$. In early 2013 beam operation was stopped for a 2-year long shutdown (LS1) to consolidate the magnet interconnection in view of reaching the design beam energy.

Beam operation resumed in 2015 at $6.5 \mathrm{TeV}$ following a dipole training campaign that took place at the end of LS1 [2]. The LHC experiments expressed a strong preference for beams with 25 ns bunch spacing, as opposed to the 50 ns spacing used in 2011-2012, as this would result in a too high number of inelastic collisions per crossing (pile-up). On the machine side 25 ns beams pose additional challenges. Given the number of new territories had to be explored, 2015 became a learning year dedicated to preparing the machine for full luminosity production in 2016-2018, with the aim of collecting over $100 \mathrm{fb}^{-1}$ until the end of 2018 .

\section{Luminosity and LHC parameters}

The event rate $d N / d t$ of a physical process with a cross-section $\sigma_{p}$ is proportional to the collider luminosity $\mathscr{L}$

$$
\frac{d N}{d t}=\mathscr{L} \sigma_{p}
$$

that can be expressed in terms of machine and beam parameters as [3]

$$
\mathscr{L}=\frac{k N^{2} f}{4 \pi \sigma_{x}^{*} \sigma_{y}^{*}} F=\frac{k N^{2} f \gamma}{4 \pi \beta^{*} \varepsilon} F
$$

Here $k$ is the number of colliding bunch pairs, $N$ the particle population of each bunch, $f=$ $11.25 \mathrm{kHz}$ is the LHC revolution frequency. For round beams at the interaction point (IP) the beam sizes in the horizontal and vertical plane $\sigma_{x}^{*}$ and $\sigma_{y}^{*}$ are identical, and $\sigma_{x}^{*} \sigma_{y}^{*}=\beta^{*} \varepsilon / \gamma$ where $\beta^{*}$ is the betatron function at the interaction point (IP), $\varepsilon$ is the normalized emittance (independent of energy) and $\gamma$ is the usual relativistic factor. $F(\leq 1)$ is a reduction factor to account for geometric luminosity reductions due to the presence of crossing angles at the IP.

The proton beam parameters are defined by the LHC injector chain. The minimum bunch spacing of $25 \mathrm{~ns}$ defines the maximum value $k=2808$. The bunch intensity is limited to $\approx$ $2-3 \times 10^{11} \mathrm{ppb}$ for isolated single bunches and to $\approx 1.3 \times 10^{11} \mathrm{ppb}$ for $25 \mathrm{~ns}$ bunch spacing, while the beam emittances range between $1 \mu \mathrm{m}$ and $3.5 \mu \mathrm{m}$.

To avoid encounters in the roughly $100 \mathrm{~m}$ long vacuum chamber that is shared by both beams around each experiments, a crossing angle is introduced at the collision point. Depending on 
bunch intensity, bunch spacing and energy, the full crossing angle varies between 200 to $400 \mu \mathrm{rad}$ for the two high luminosity experiments. The minimum separation between the beams should correspond to $\approx 9-10$ beam sizes to avoid issues with the long range beam-beam interactions [4]. A consequence of the crossing angle is a reduction of the luminosity due to the geometric overlap of the beams, $F \sim 0.60$ in 2018 .

The minimum value of $\beta^{*}$ is defined by the mechanical aperture of the quadrupoles around the IPs, the crossing angle and the required margin between the beam halo and the aperture. $\beta^{*}$ could be lowered progressively over the years as the understanding of the LHC machine improved.

\section{Overview of LHC Run 2}

\subsection{Operation in 2015}

The year 2015 was dedicated to establishing operation at $6.5 \mathrm{TeV}$ per beam and with standard 25 ns bunch spacing [1]. The first three months were dedicated to magnet powering tests and the magnet training campaign to establish a reliable and reproducible magnet performance at magnetic fields equivalent to $6.5 \mathrm{TeV}$ beam energy. The beam commissioning was accomplished using the $50 \mathrm{~ns}$ bunch spacing to avoid electron cloud effects during this initial period. By mid-July 2017, following an electron cloud scrubbing run, operation switched to $25 \mathrm{~ns}$ bunch spacing, initially with a reduced number of bunches to limit the total intensity and stored energy. The beam intensity was ramped up until the end of the year by increasing step-wise the number of bunches injected to 2244 bunches per beam. Despite the prolonged periods of e-cloud scrubbing, the intensity ramp up was mostly limited by the heat load induced on the cryogenic system [5].

\subsection{Operation in 2016}

In 2016 only 4 weeks were required for the beam commissioning that was directly followed by an intensity ramp up and luminosity production using the standard $25 \mathrm{~ns}$ bunch spacing. From Fig. 1 one can perceive that this was also the first year with substantial luminosity production. On 26 June the LHC attained its design luminosity of $1 \times 10^{34} \mathrm{~cm}^{-2} \mathrm{~s}^{-1}$. In parallel, the injector chain prepared a high brightness $25 \mathrm{~ns}$ beam, based on a Batch Compression Merging and Splitting (BCMS) scheme [6]. The LHC used this beam successfully for production, resulting in a transverse emittance at the start of collisions of only $2 \mu \mathrm{m}$. In combination with a reduction of the half crossing angle from $185 \mu \mathrm{rad}$ to $140 \mu \mathrm{rad}$ in September led to a further increase of the peak luminosity, as can be observed in Fig. 1, with a record peak luminosity of $1.4 \times 10^{34} \mathrm{~cm}^{-2} \mathrm{~s}^{-1}$. In August an intermittent inter-turn short circuit was observed in one of the dipole magnets in half cell 31 left of IP2 (31L2) that is part of Sector 1-2, one of the eight sectors that constitute the LHC. Despite this issue, luminosity production continued, but the decision was taken to replace the magnet during the upcoming winter shutdown. The proton physics run ended on a very positive note with $40 \mathrm{fb}^{-1}$ of integrated luminosity for ATLAS and CMS. The LHC continued running successfully for another 4 weeks with proton lead collisions.

\subsection{Operation in 2017}

Following the magnet replacement in Sector 1-2, requiring warming up and cooling down the 


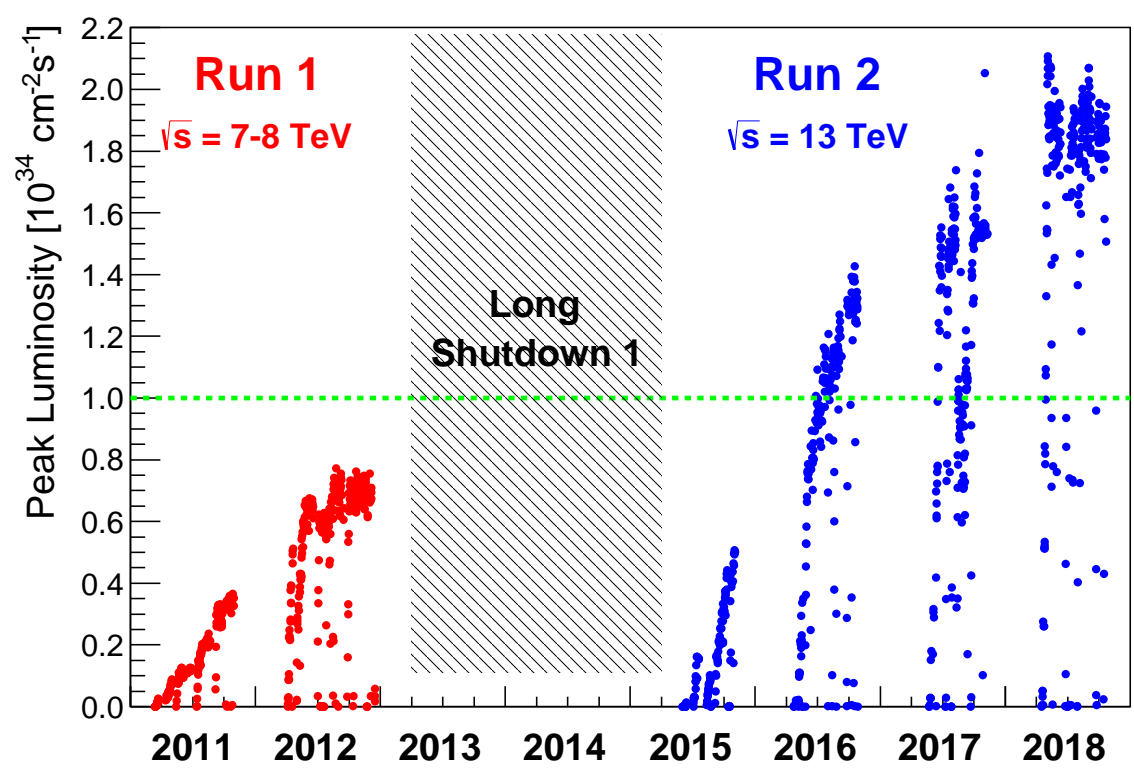

Figure 1: Evolution of the LHC peak luminosity (ATLAS/CMS experiments) between 2011 and 2018. The green dashed line represents the design luminosity.

$3 \mathrm{~km}$-long sector, the re-commissioning of the circuits included a long list of additional tests to be performed on the sector that underwent the thermal cycle.

The first beam was injected on 29 April and first collisions with a few bunches were established 4 weeks later. Subsequently a period with interleaved commissioning and intensity ramp up followed. Before reaching 2556 bunches in stable beams in July a one-week scrubbing run was performed to reduce the secondary electron emission yield of the beam screen, hence the production of electron clouds in the sector that had been warmed up.

Already during beam commissioning, abnormal and sudden beam losses, some leading to beam dumps, were observed in the beam vacuum for both beams at the level of a magnet interconnect of half-cell 16 left of IP2 (16L2). It was realized much later that these losses were induced by an accidental inlet of air into the beam vacuum with the beam screen at $20 \mathrm{~K}$, following the magnet replacement [7, 8]. In August a beam screen flushing was attempted where the beam screen is warmed up from its usual $20 \mathrm{~K}$ to $80 \mathrm{~K}$ with the aim to evaporate frozen gas and condensate it on the surrounding cold bore, out of the sight of the beam. Unfortunately this operation did not succeed. Since the loss mechanism was suspected to be influenced by electron cloud, the LHC injector chain produced the $25 \mathrm{~ns} 8 \mathrm{~b} 4 \mathrm{e}$ beam that was used in the LHC as of September. The 8b4e beam structure consists of eight bunches spaced by $25 \mathrm{~ns}$ followed by four empty buckets. This results in 1916 bunches per beam and suppresses the electron cloud production drastically thanks to the many empty slots in the beam. Once proven successful in mitigating the $16 \mathrm{~L} 2$ issue, this scheme was further enhanced in the injectors. The main beam parameters for these beams that allowed efficient luminosity production, despite the 16L2 issue, are given in Table 1.

Following further measurements and studies on the available aperture it was decided to re- 


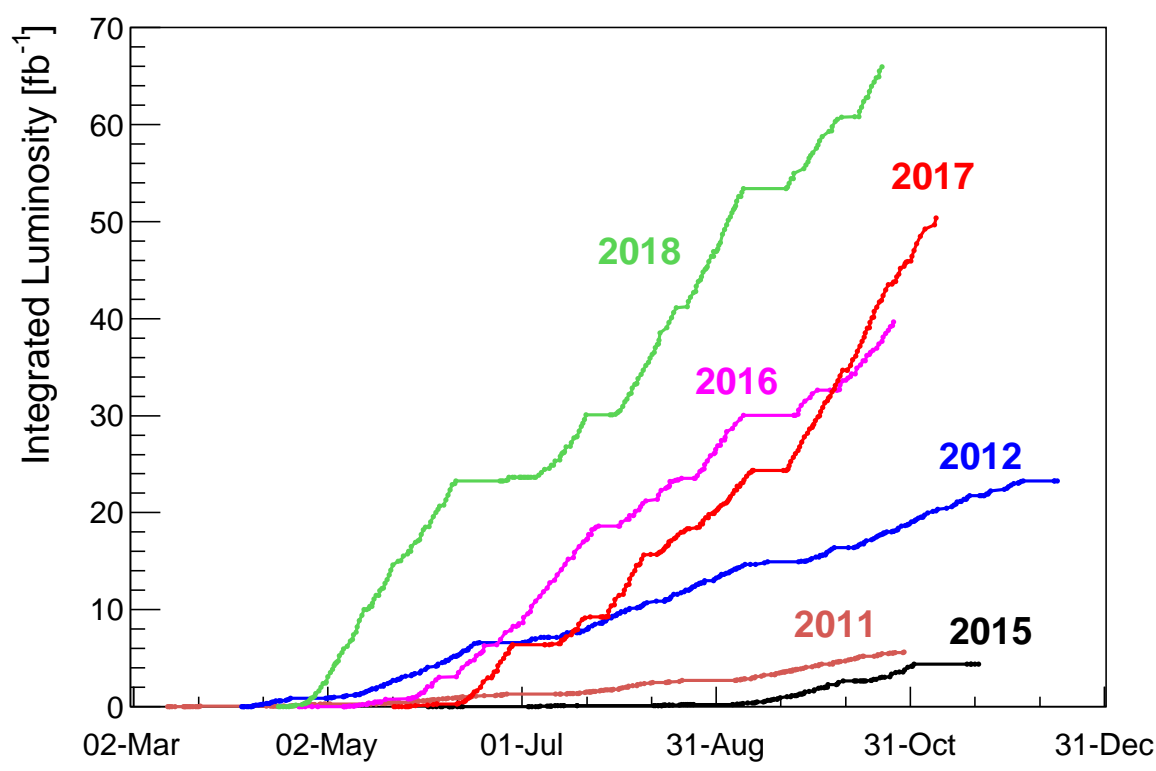

Figure 2: Evolution of the yearly LHC integrated luminosity (ATLAS/CMS experiments) between 2011 and 2018, with $66 \mathrm{fb}^{-1}$ delivered in 2018 .

duce the $\beta$ tron-function at IP1 and IP5 from $40 \mathrm{~cm}$ to $30 \mathrm{~cm}$. This resulted on 2 November in a new luminosity record of $2.06 \times 10^{34} \mathrm{~cm}^{-2} \mathrm{~s}^{-1}$. The number of inelastic collisions per bunch crossing (pile-up) in the experiments ATLAS and CMS was beyond the acceptable, consequently the instantaneous luminosity was levelled to $1.5 \times 10^{34} \mathrm{~cm}^{-2} \mathrm{~s}^{-1}$, using levelling by beam separation. The 2017 proton physics run that was hampered by the $16 \mathrm{~L} 2$ issue nevertheless ended with a record integrated luminosity of $50 \mathrm{fb}^{-1}$.

\subsection{Operation in 2018}

To resolve the $16 \mathrm{~L} 2$ issue sector 1-2 was warmed up to $90 \mathrm{~K}$ during the $2017-2018$ winter shutdown, allowing the evacuation of about 7 litres of gasses like oxygen and nitrogen, but not of the water vapour which was estimated to be 0.1 gram per beam vacuum [7].

The first beam was injected on 30 April and first collisions with a few bunches were established 3 weeks later. Subsequently, a period with interleaved commissioning and intensity ramp up followed, with the maximum of 2556 bunches achieved early May, two weeks ahead of schedule. During the intensity ramp-up, beam losses induced by 16L2, although much lower than in 2017, where present and closely monitored. These beam losses are of two types: firstly a steady-state or constant beam loss that depends on the total number of particles per beam. This beam loss is mitigated by a special solenoid that was installed during the second half of 2017 [7]. Secondly, erratic beam loss spikes that add to the steady-state losses, potentially surpassing the dump threshold. These spikes were "conditioned away", allowing running with the 2556-bunch BCMS beam in 2018, despite some occasional beam dumps. The 2018 proton physics run ended on 24 October and accumulated $66 \mathrm{fb}^{-1}$ of integrated luminosity for ATLAS and CMS. 
The year ended with a 24-day lead-lead ion run at $6.37 \mathrm{TeV}$ beam energy.

\section{Performance overview}

The LHC machine and beam parameters are summarised in Table 1. The LHC machine availability for stable collisions was 35\% in 2015 and 50\% between 2016 and 2018. Fig. 1 presents the increase of peak luminosity over the years, but also the steepening slope of the luminosity ramp up between 2015 and 2018, indicating efficient re-commissioning and fast intensity ramp up. The design luminosity of $1 \times 10^{34} \mathrm{~cm}^{-2} \mathrm{~s}^{-1}$ is indicated by the green line and was passed for the first time in 2016. The LHC peak luminosity at $6.5 \mathrm{TeV}$ is limited to $\approx 2.2 \times 10^{34} \mathrm{~cm}^{-2} \mathrm{~s}^{-1}$ by the cryogenic cooling capacity of the LHC interaction region quadrupoles. The yearly integrated luminosity plot for the years 2011 to 2018 is given in Fig. 2. One can clearly distinguish the commissioning years 2011 (Run 1) and 2015 (Run 2) from the production years 2012, 2016, 2017 and 2018. The integrated luminosity evolution is presented in Fig. 3, the total integrated luminosity reached over $160 \mathrm{fb}^{-1}$ for Run 2 and almost $200 \mathrm{fb}^{-1}$ for the total 2010-2018.

Table 1: Beam and machine parameters for collisions in 2012, 2016, 2017 and 2018 compared to the design.

\begin{tabular}{lccccc} 
Parameter & Design & 2012 & 2016 & 2017 & 2018 \\
\hline beam energy [TeV] & 7 & 4 & 6.5 & 6.5 & 6.5 \\
bunch spacing [ns] & 25 & 50 & 25 & 25 & 25 \\
$\beta^{*}$ CMS/ATLAS [cm] & 55 & 60 & 40 & $40 / 30$ & $30-25$ \\
crossing angle $[\mu \mathrm{rad}]$ & 285 & 290 & $370 / 280$ & $300-240$ & $320-260$ \\
bunch population $N\left[10^{11} \mathrm{ppb}\right]$ & 1.15 & 1.65 & 1.1 & 1.15 & 1.15 \\
normalized emittance $\varepsilon[\mu \mathrm{m}]$ & 3.75 & 2.5 & 2.2 & 2.2 & 2.0 \\
number of bunches per ring $k$ & 2808 & 1374 & 2220 & 2556 & 2556 \\
peak luminosity L $\left[10^{34} \mathrm{~cm}^{-2} \mathrm{~s}^{-1}\right]$ & 1 & 0.75 & 1.4 & 2.05 & 2.01 \\
peak average event pile-up $\mu$ & $\sim 20$ & $\sim 35$ & $\sim 50$ & $\sim 55$ & $\sim 60$ \\
peak stored energy $[\mathrm{MJ}]$ & 360 & 145 & 270 & 320 & 340
\end{tabular}

\section{Performance challenges}

\subsection{Electron clouds}

Since the start of LHC operation with bunch trains electon clouds (e-clouds) form one of the main performance limitations for the LHC. These e-clouds cause transverse emittance blow up and are able to trigger beam instabilities, causing beam losses. In addition their production puts a large constraint on the cryogenic system as they form a major source of heat load on the beam screen [9]. For a reason that is not understood four LHC arc section present low e-ecloud activity and heat load, while four others exhibit high activity, with up to twice higher heat load. The production of e-cloud is very sensitive to the secondary electron emission yield of the beam screen surface. Experience has shown that the surface of the beam screen can be conditioned by exposing the surface for prolonged period to high rates of e-cloud. In practice, at the start of a yearly run and once the LHC is sufficiently commissioned to house a large number of bunches at low energy, a scrubbing run ois 


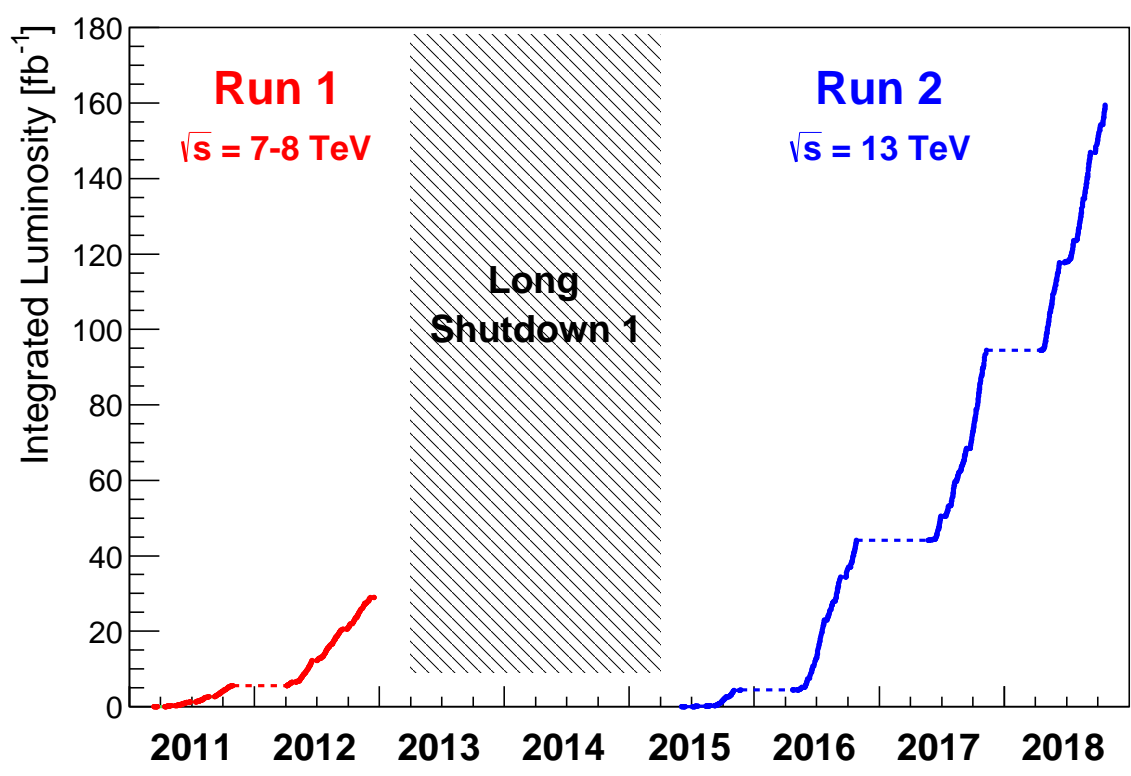

Figure 3: Evolution of the LHC integrated luminosity (ATLAS/CMS experiments) between 2011 and 2018 , $30 \mathrm{fb}^{-1}$ have been delivered in Run 1 and just over $160 \mathrm{fb}^{-1}$ in Run 2

scheduled to re-establish conditions that allow to accelerate safely a full machine to high energy for collisions. The duration ranges from $1-2$ days (no intervention on the vacuum) to around one week when large sectors of the vacuum system were exposed to air.

\subsection{Fast beam loss events}

Fast loss events, nicknamed Unidentified Falling Objects (UFOs), have been observed at the LHC since 2010 [10]. The loss duration is in the millisecond time range, and UFOs are believed to be due to dust particles falling in the vacuum chamber and interacting with the beam, creating particle showers that deposit energy in the magnets and that are then detected by the Beam Loss Monitors (BLM). They may affect machine availability, as the most intense ones can trigger a beam dump by the BLM system, or initiate a magnet quench.

Following the long shutdown UFO rates in 2015 were as high as 30-40 events per hour, and decreased with beam time (conditioning) to $\approx 10$ events per hour [11]. They caused 22 beam dumps including 3 beam-induced quenches in 2015. The initial strategy was to prevent if possible all UFO-induced magnet quenches [12], but it was realized in 2015 that most of the UFOs events leading to beam dumps would not have caused quenches. Thus the policy changed and the BLM thresholds were increased to allow a few UFO-induced quenches per a year. A further increase was put in place in 2016, while the UFO rates presented continued to come down to around 2 UFOs per hour [13]. The dump numbers decreased to around 5 dumps in 2018.

\subsection{Luminosity levelling}

Luminosity levelling is generally applied to reduce the number of inelastic collisions per bunch crossing when the instantaneous luminosity is too high. This has been done routinely for the two 


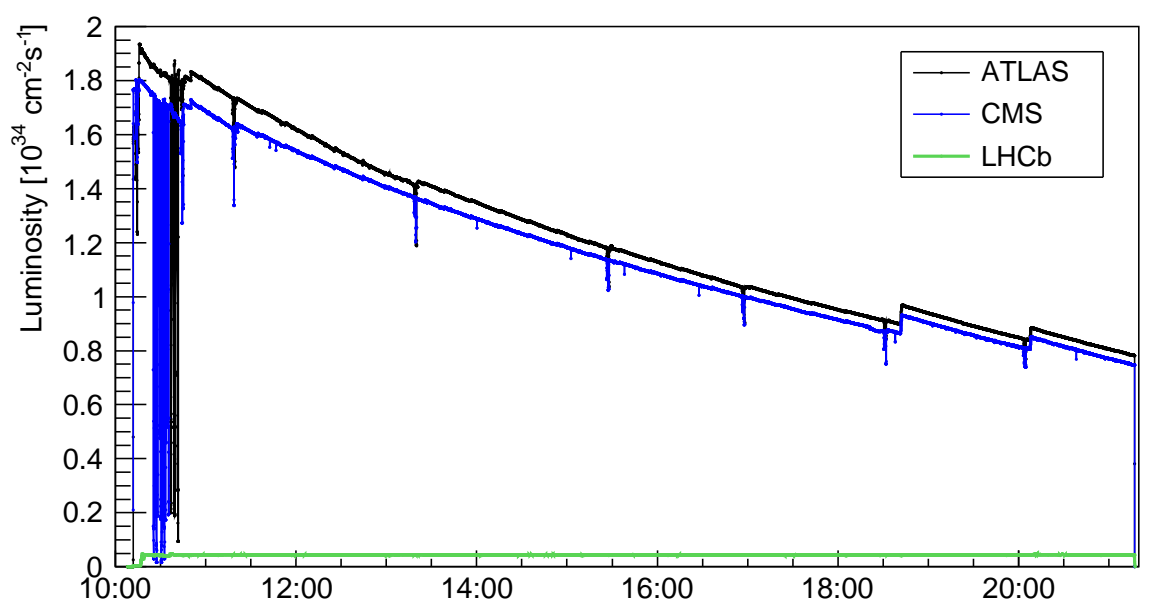

Figure 4: Example for the luminosity evolution of the ATLAS, CMS and LHCb experiments in a typical fill of the 2018 run. The luminosity of LHCb is levelled by beam separation. The upward steps of the ATLAS and CMS luminosities in the second half of the fill are due to $\beta^{*}$ levelling.

low luminosity experiments ALICE and LHCb since 2011 to maintain a stable low luminosity. When the achieved peak luminosity also exceeded the pile-up limit of ATLAS and CMS in 2017, levelling by beam separation was applied for those experiments.

As a result of luminosity burn-off during collisions the crossing angles and $\beta$-functions can be reduced during the fill, increasing the instantaneous luminosity $[14,15]$. This anti-levelling scheme has been developed, tested and validated during dedicated machine development (MD) sessions and deployed in steps. In the second half of 2017 the anti-levelling by reducing the half crossing angle from $150 \mu \mathrm{rad}$ in three steps to $120 \mu \mathrm{rad}$ was deployed operationally. In 2018 the steps were removed and a continuous crossing angle anti-levelling, based on the dynamic aperture evolution is used. In addition $\beta *$ anti-levelling was added to reduce the $\beta *^{*}$ from $30 \mathrm{~cm}$ to $25 \mathrm{~cm}$ in two steps, see Fig. 4. Both anti-levelling schemes increase the luminosity production with a few percent, but the gain in operational experience is also very important for the the upcoming Run 3 and the LHC high luminosity upgrade (HL-LHC) [16].

\section{Outlook}

Following the upcoming long shutdown 2019-2020, the LHC will be prepared to operation at $7 \mathrm{TeV}$ beam energy. This expected to require a two to three month long training quench campaign with over 400 training quenches of the main dipole magnets. During the same shutdown the LHC injectors will undergo a major upgrade to push the bunch intensity in the LHC to $1.8 \times 10^{11}$ protons per bunch for the years 2022-2023. With such high bunch currents it will be possible to level the LHC luminosity around $2 \times 10^{34} \mathrm{~cm}^{-2} \mathrm{~s}^{-1}$ for 8 to 20 hours depending on the exact machine configuration. This opens a window to produce $100 \mathrm{fb}^{-1}$ per year by 2023 . A detailled study is underway, a report on operation during this Run 2 is in preparation. 


\section{References}

[1] O. S. Brüning et al. (Eds), LHC Design Report, CERN, Geneva, Switzerland, Rep. CERN-2004-003-V-1, 2004.

[2] A. Verweij, Circuit Performance at 6.5 TeV and beyond, LHC Performance Workshop, Chamonix, France, 2016.

[3] B. Muratori, Luminosity and luminous region calculations for the LHC, CERN, Geneva, Switzerland, Rep. LHC Project Note 301, 2002.

[4] M. Crouch et al., The Long-Range Beam-Beam Limit in the LHC, presented at IPAC17, Copenhagen, Denmark, May 2017, paper TUPVA025, this conference.

[5] G. Ferlin et al., Cryogenics, LHC Performance Workshop, Chamonix, France (2016).

[6] H. Damerau et al., RF manipulations for higher brightness LHC-type beams, in Proceedings of IPAC2013, Shanghai, China, paper WEPEA044.

[7] J.M. Jiménez at al., Observations, Analysis and Mitigation of Recurrent LHC Beam Dumps Caused by Fast Losses in Arc Half-Cell 16L2, in Proceedings of IPAC2018, Vancouver, Canada, paper MOPMF053.

[8] B.Salvant et al., Experimental Characterisation of a Fast Instability Linked to Losses in the 16L2 Cryogenic Half-Cell in the CERN LHC, in Proceedings of IPAC2018, Vancouver, Canada, paper THPAF058.

[9] G. Rumolo et al., Electron Cloud at the LHC and LHC Injectors, in Proc. of IPAC2017, Copenhagen, Danmark, paper MOZA1

[10] T. Baer et al, UFOs in the LHC, presented at IPAC11, San Sebastian, Spain, September 2011, paper TUPC137.

[11] G. Papotti et al, Macroparticle-Induced Losses During 6.5 TeV LHC Operation, presented at IPAC16, Busan, Korea, May 2016, paper TUPMW023.

[12] A. Lechner et al, BLM thresholds for post-LS1 LHC operation: UFOs and orbit bumps in the arcs and straight sections, Proc. of the Workshop on Beam Induced Quenches, CERN, Geneva, Switzerland, 2014.

[13] A. Lechner, Expected impact of 7 TeV operation on LHC availability, LHC Performance Workshop 2017, Chamonix, France, 2017, https://indico.cern.ch/event/580313.

[14] M. Hostettler at al., Online Luminosity Control and Steering at the LHC, in Proc. of ICALEPC2017, Barcelona, Spain, paper TUSH201.

[15] M. Hostettler et al. $\beta *$ Levelling Using the LHC Lumi Server (MD 2427), CERN-ACC-NOTE-2018-0001

[16] G. Apollinari, I. Béjar Alonso, O. Brünning, P. Fessia, M. Lamont, L. Rossi, L. Tavia (Eds) High-Luminosity Large Hadron Collider (HL-LHC) Technical Design Report, CERN-2017-007-M. 